\title{
Des mutations du proto-oncogène RET dans la maladie de Hirschsprung: un gène à tout faire!
}

La maladie de Hirschsprung est une des plus fréquentes malformations digestives puisqu'elle touche un enfant sur 5000 naissances [1]. Classiquement, elle entraîne une occlusion intestinale néonatale sévère conduisant à un traitement chirurgical qui garantit actuellement un pronostic favorable pour la plupart des enfants atteints. Cette occlusion a pour origine l'absence du système nerveux autonome de l'intestin terminal qui est formé de cellules ganglionnaires ayant pour origine les crêtes neurales au niveau des somites 1 à $7[2,3]$. Il s'agit donc d'une neurocristopathie [4].

Bien que la grande majorité des cas soient sporadiques, plusieurs arguments militaient de longue date en faveur du rôle de facteurs génétiques dans la survenue de la maladie de Hirschsprung [5]. En particulier, les analyses de ségrégation dans de grandes séries proposaient un modèle multifactoriel duquel se dégageait un gène autosomique dominant de faible pénétrance responsable des formes longues de la maladie (moins de $20 \%$ des cas [6]).

Dans le numéro de août/septembre 1993 de médecine sciences [7], nous rapportions la localisation d'un tel gène dominant (locus HSCR) entre les loci D10S208 et D10S196 génétiquement et physiquement cartographiés dans la région proximale du bras long du chromosome 10 (locus $\operatorname{HSCR}[8,9])$. Cette information constituait un premier pas vers le démembrement génétique de cette maladie, mais elle offrait aussi la possibilité d'entreprendre l'étude moléculaire de la région $10 \mathrm{q} 11.2$ à la recherche du gène HSCR. Le clonage positionnel de ce gène par cartographie physique et recherche de séquences exprimées semblait une tâche insurmontable du fait de la grande taille de la région désignée ( 10 centimorgans) et du caractère exceptionnel des remaniements chromosomiques. De la même manière, le petit nombre de familles connues et la faible pénétrance de ce gène $(0,51$ chez les filles, 0,66 chez les garçons) rendaient très hasardeuse la cartographie génétique fine de cette région.

En revanche, plusieurs gènes d'intérêt se trouvaient déjà localisés dans la région proximale du chromosome 10 et, particulièrement, le protooncogène RET de la famille des récepteurs tyrosine kinase membranaires [10]. Plusieurs arguments nous ont incités à considérer ce gène comme un candidat majeur pour la maladie de Hirschsprung. - Le proto-oncogène RET code pour deux isoformes (par épissage alternatif) dont ni le ligand, ni la fonction ne sont connus. Pourtant, des formes remaniées de cet oncogène sont fortement exprimées dans des tumeurs ayant pour origine des cellules de la crête neurale: neuroblastomes, phéochromocytomes et carcinomes médullaires de la thyroïde [11]. Quant aux territoires d'expression de RET dans des tissus normaux, ils sont eux aussi restreints à des lignées dérivées de la crête neurale comme les cellules Schwann, certaines cellules cérébelleuses, la médullosurrénale et les ganglions sympathiques [12]. Ces observations sont à mettre en parallèle avec l'origine neurale du système nerveux entérique.

- La localisation physique du gène RET le plaçait précisément dans l'intervalle génétique que nous avions défini comme intervalle can- didat pour le gène de la maladie de Hirschsprung. De surcroît, le gène RET est délété chez deux enfants présentant une maladie de Hirschsprung de forme longue associée à un remaniement chromosomique cytogénétiquement visible.

- Enfin, nos toutes dernières données de cartographie génétique et celles d'un groupe italien faisaient du locus RET le locus polymorphe de la région le plus voisin du locus HSCR 1 (lod score en bi-point de 7,54 dans notre série, sans aucune recombinaison). Cette dernière observation était très convaincante, car elle rendait indistingable génétiquement le locus RET et le locus $H S C R[13,14]$.

Devant ces arguments, plusieurs groupes se sont lancés à la recherche de mutations de RET chez des patients atteints de maladie de Hirschsprung. Les résultats viennent d'être publiés dans la revue Nature et montrent qu'en effet des mutations ponctuelles des domaines extra-cellulaires ou tyrosine kinase de RET sont associés à la maladie de Hirschsprung dans des cas familiaux (figure $1[15,16]$ ). Pour notre part, des analyses préliminaires ont indiqué qu'aucune délétion ou réarrangement important n'ont été retrouvés dans notre série de formes familiales dont on avait fait la preuve que le gène était lié au chromosome 10q11. Dès lors, notre stratégie a consisté à analyser la séquence codante de RET à la recherche d'anomalies plus subtiles ou de mutations ponctuelles en combinant l'étude du polymorphisme de conformation des simples brins d'ADN (SSCP) [17] et le séquençage direct des exons de RET lorsque des profils anormaux de 


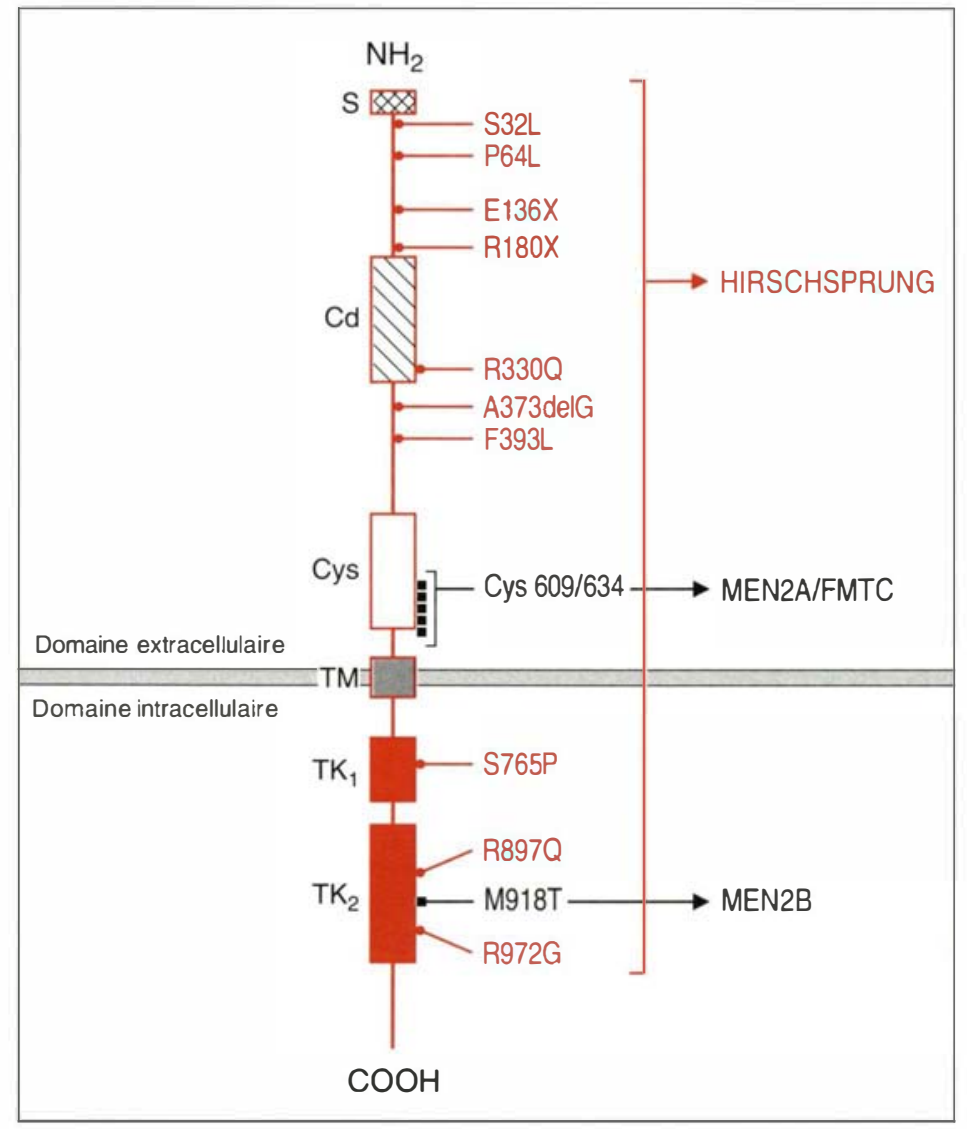

Figure 1. Protéine RET. Les 21 exons du gène RET codent pour un récepteur tyrosine kinase membranaire dont le domaine extracellulaire aminoterminal comporte une séquence signal (S), une région d'homologie avec les cadhérines $(C d)$ et des sites prédictifs de liaison du calcium (barres) et une région juxtatransmembranaire riche en cystéines (Cys). Le domaine transmembranaire (TM) de RET est codé intégralement par l'exon 11. On trouve enfin dans la région intracellulaire deux domaines tyrosine kinases (TK1 et 2). Les mutations ponctuelles de RET et les substitutions d'acides aminés que l'on peut en prédire sont indiquées en rouge pour la maladie de Hirschsprung [15, 16] et en noir pour MEN2A [22-24], les formes familiales de cancer médullaire de la thyroïde (FMTC [23, 24]) et MEN2B[25, 26]. Les mutations associées à MEN2A touchent exclusivement 5 codons cystéine des exons 10 et 11 (codons 609, 611, 618, 620 et 634 [24]). Code à une lettre des acides aminés: $A: A l a ; C: C y s ; D: A s p$; $E$ : Glu; F: Phe; G: Gly; H: His ; I: Ile; K: Lys ; L: Leu; M: Met; N: Asn; $P$ : Pro; $Q$ : Gln; $R$ : Arg; S: Ser; T: Thr; V: Val; W: Trp; Y:Tyr.
SSCP ont été observés. Nous avons d'abord entrepris l'analyse de neuf exons codant pour les domaines extracellulaires ou transmembranaires de la protéine. Rapidement, des profils de migration anormaux en SSCP ont été trouvés, suggérant des variations de séquences nucléotidiques dans les exons $2,3,5$ et 6 chez les cas index de six familles de Hirschsprung. L'analyse directe de la séquence de ces six patients a montré leur hétérozygotie pour des mutations faux-sens ou non-sens permettant de prédire, soit des substitutions d'acides aminés dans la protéine, soit la terminaison prématurée de la traduction de l'ARNm RET. Tâchant de réunir d'autres éléments en faveur de la responsabilité de ces mutations dans la maladie de Hirschsprung, nous avons montré que les mutations de RET coségrègent régulièrement avec la maladie chez les individus atteints et les por- teurs obligatoires au sein de cinq familles dans lesquelles cette étude a été possible, mais qu'elles sont absentes dans une série de quarantecinq témoins $(p<0,001)$.

Plusieurs arguments suggèrent que les mutations de $R E T$ dans la maladie de Hirschsprung conduisent à l'absence d'expression de ce gène ou à l'inactivation de son produit à l'état hétérozygote. Deux délétions de la région proximale du bras long du chromosome 10 ont été rapportées en association avec la maladie de Hirschsprung et, dans les deux cas, la délétion comprend le locus $R E T$. Les deux mutations non-sens identifiées sont des codons stop très précoces dont on peut prédire qu'ils produisent une protéine largement tronquée et très probablement non fonctionnelle. Quant aux mutations faux-sens de RET chez des patients atteints de maladie de Hirschsprung, elles concernent des acides aminés très conservés entre homme et souris et sont réparties de façon apparemment aléatoire dans le domaine de liaison vraisemblable de $R E T$ à son ligand, ce qui est compatible avec la diversité des événements habituellement associés à l'inactivation d'un gène. Dans le même ordre d'idées, une des six mutations identifiées se trouve dans l'exon 5 du gène de RET qui code pour un domaine ayant une forte homologie avec la famille des cadhérines, des molécules impliquées dans l'adhérence cellule/cellule (figure $1[18$, 19]). La disparition des interactions cellulaires modulées par ce motif est un modèle séduisant permettant peut-être d'expliquer le défaut de migration des cellules de la crête neurale observé dans la maladie de Hirschsprung [2, 3].

Pour finir, un troisième article de ce même numéro de Nature rapporte les résultats de l'invalidation (par 
insertion) du gène ret chez la souris qui (à l'état homozygote) conduit à l'absence de système nerveux autonome intestinal [20]. La mariée est trop belle !

Cependant, ces observations posent certainement plus de questions qu'elles n'en résolvent:

- Bien qu'un ensemble de plus de vingt familles de maladie de Hirschsprung étudiées dans notre série soit apparemment lié au chromosome 10q11.2 (Edery et al., soumis), les mutations de RET n'ont été pour l'instant détectées que chez cinq d'entre elles. Naturellement, cela peut être dû à l'existence de mutations dans d'autres domaines de ce proto-oncogène (seulement $56 \%$ de la séquence codante ont été étudiés pour l'instant), à des mutations introniques ou encore à des mutations promotrices. Cependant, certaines de nos données préliminaires laissent envisager d'autres gènes jouant un rôle dans la maladie de Hirschsprung. Tout compte fait, cela rendrait mieux compte de l'existence d'au moins trois modèles murins de mégacolon congénital, ségrégeant selon un mode d'hérédité monofactorielle [21], et de la coexistence indéniable de la maladie de Hirschsprung avec des anomalies d'autres chromosomes, notamment des délétions interstitielles du bras long du chromosome 13 ou la trisomie 21 .

- Qu'en est-il des cas sporadiques? Ils représentent plus de $80 \%$ des maladies de Hirschsprung, surtout dans sa forme classique où la zone aganglionique ne dépasse pas le recto-sigmoïde. Bien entendu, il peut s'agir de formes "familiales" masquées par des défauts de pénétrance ou une expression très faible de la maladie chez les parents. Dès lors, il est envisageable de trouver des mutations de RET chez de tels patients. Mais, il est aussi possible que le modèle multifactoriel trouve ici vraiment sa place en combinant l'effet d'un gène majeur (RET) avec l'action d'un ou de plusieurs autres gènes modificateurs, rendant compte alors de ces cas isolés. de RET dans la maladie de Hirschsprung? Nos données sont très en faveur de mutations dans le domaine extracellulaire. Cependant, le groupe italien de Gênes qui publie dans le même numéro de Nature a trouvé des mutations du domaine tyrosine kinase intracytoplasmique de RET (figure 1[16]). Une des priorités est donc d'effectuer une cartographie détaillée des mutations de RET dans les formes familiales de maladie de Hirschsprung, ce qui devrait permettre d'approcher, si ce n'est de comprendre, les relations qui existent entre les anomalies de ce gène et le phénotype observé.

- Pour finir, un des éléments les plus frappants est le fait que des mutations de RET ont déjà été identifiées comme la cause d'un tout autre groupe de maladies, les endocrinopathies néoplasiques multiples de type 2A (MEN2A) qui sont des syndromes de prédisposition héréditaire aux cancers des cellules endocrines dérivées de la crête neurale : phéochromocytomes, carcinomes médullaires de la thyroïde ou adénomes des parathyroïdes [22, 23]. A l'inverse des mutations Hirschsprung, les mutations ponctuelles du gène RET retrouvées dans MEN2A sont exclusivement restreintes à cinq codons cystéine du domaine extracellulaire juxtamembranaire qui code pour une région de la protéine très riche en ce type d'acides aminés (figure 1 [24]). Pour achever ce tableau complexe, Buyhs et al. rapportent dans le même numéro de Nature une mutation ponctuelle de RET responsable de MEN2B, une forme sévère de ces endocrinopathies néoplasiques, souvent associées à des ganglioneuromes (figure 1 [25, 26]). Ajoutons que la mutation rapportée dans ce cas ne porte pas sur une cystéine mais qu'elle est située dans le domaine tyrosine kinase!

Plus que l'implication d'un même gène dans deux maladies, comme c'est le cas pour le gène CFTR (mucoviscidose ou agénésie des canaux déférents) ou encore le gène récepteur des androgènes (atrophie bulbaire et spinale de
Kennedy ou testicule féminisant), c'est l'opposition des deux phénotypes observés pour des mutations ponctuelles de RET qui surprend: d'une part, des mutations probablement inactivatrices, conduisant à des anomalies précoces du développement embryonnaire dans la maladie de Hirschsprung et, d'autre part, des mutations vraisemblablement activatrices, conduisant à des cancers héréditaires des dérivés de la crête neurale chez les patients atteints de MEN2A, MEN2B ou de carcinomes médullaires de la thyroïde. Ainsi, les mutations germinales du gène $R E T$ offrent-elles le modèle intriguant d'un récepteur tyrosine kinase membranaire dont les mutations peuvent conduire aux deux extrêmes des anomalies de contrôle du développement cellulaire: une embryopathie et un cancer

\section{RÉFÉRENCES}

1. Hirschsprung H. Stuhltragheit neugeborener infolge von dilatation und hypertrophic des colons. Ib Kinderheilk 1887; 27 : 1-27.

2. Okamato E, Ueda T. Embryogenesis of the intramural ganglia of the gut and its relation to Hirschsprung's disease. / Pediatr Surg 1967; 2: 437-43.

3. Le Douarin N, Teillet MA. The migration of neural crest cells to the wall of the digestive tract in avian embryo. / Embryol Exp Morphol 1973; 30 : 31-48.

4. Bolande RP. The neurocristopathies; a unifying concept of disease arising in neural crest maldevelopment. Hum Pathol 1973 ; 5 : 409-29.

5. Bodian M, Carter CO. A family study of Hirschsprung disease. Ann Hum Genet $1963 ; 26: 261-77$

6. Badner JA, Sieber WK, (jarver KL, Chakravarti A. A genetic study of Hirschsprung disease. Am J Hum Genet 1990; 46 : 568-80.

7. Lyonnet S, Edery P, Nihoul-Fékété C, Munnich $\mathrm{A}$. Un gène de la maladie de Hirschsprung localisé sur le bras long du chromosome 10: une brèche dans le modèle multif actoriel des malformations de l'enfant. médecine/sciences 1993; 9: 994-5.

8. Lyonnet S, Bolino A, Pelet A, Abel L, Nihoul-Fékété C, Briard ML, Mok-Siu V, Kaariainen H, Martucciello G, Lerone M, 
Puliti A, Luo Y, W'eissenbach J, Devoto M, Munnich A, Romeo G. A gene for Hirschs prung disease maps to the proximal long arm of chromosome 10. Nature (jent 1993; $4: 346-50$

9. Angrist M, Kauffman E, Slaugenhaupt SA, Matise TC, Puffenberger E(; W'ashington SS, Lipson A, Cass DT, Eyna TR, Weeks IDE, Sieber W', Chakravarti A. A gene for Hirschsprung disease (megacolon) in the pericentromeric region of human chromosome 10. Nature (jenet 1993; $4: 351-6$.

10. Takahashi M, Buma Y, Iwamoto T, Inaguma $\mathrm{Y}$, Ikeda $\mathrm{H}$. Cloning and expression of the ret proto-oncogene encoding a tyrosine kinase with two potential transmembrane domains. Oncogene 1988; 3: 571-8

11. Ikeda 1, Ishizaka Y, Tahira T, Suzuki T, (Onda M, Sugimura T, Nagao M. Specific expression of the RET proto-oncogene in human neuroblastoma cell lines. Oncogene $1990 ; 5$ : 1291-6.

12. Nakamura T, Ischizaka $Y$, Nagao $M$, Hara M, Ishikawa T. Expression of the RET proto-oncogene in normal and neoplastic tissues. Oncogene (sous presse).

13. Luo Y, Ceccherini 1, Pasini B, Matera I, Bicocchi MP, Barone V, Bocciardi R, Kääriāinen $\mathrm{H}$, W'eber I), Devoto $\mathrm{M}$, Romeo (i. Close linkage with the $R E T$ protooncogene and boundaries of deletion mutations in autosomal dominant Hirschsprung disease. Hum Mol Genet 1993; 2: 1803-8.

14. Edery P, L yonnet S, Pelet A, Le Merrer $M$, Pelet $A$, Flintoff $~ W '$, Nihoul-Fékété C, Weissenbach J, Munnich A. Hirschsprung disease: mapping of a dominant gene linked to the RET lorus on the proximal long arm of chromosome 10. $\mathrm{Am} /$ Hum (ienet 1993; 53 (suppl) : 996.

15. Edery P, Lyonnet S, Mulligan L, Pelet A, Dow E, Abel I, Holder S, Nihoul-Fékété C, Ponder BAJ, Munnich A. Mutations of the RET proto-oncogene in Hirschsprung's disease. Nature 1994; 367 : 378-80.

16. Romeo G, Ronchetto P, Luo Y, Barone V, Seri M, Ceccherini I, Pasini B, Bocciardi $\mathrm{R}$, Lerone $M$, Kääriäinen $\mathrm{H}$, Martucciello G. Point mutations affecting the tyrosine kinase domain of the RET proto-oncogene in Hirschsprung's disease. Nature 1994; 367: 377-8.

17. Ireyfus JC, Akli S, Poenaru L. Maladie de Tay-Sachs et de Sandhoff: les déficits en $\beta$-hexosaminidases, modèles de maladies des lysosomes. médecine/sciences $1992 ; 8: 797-804$

18. Iwamoto $\mathrm{T}$, Taniguchi $\mathrm{M}$, Asai $\mathrm{N}$, Ohkusu K, Nakashima I, Takahashi $M$ cDNA cloning of the mouse ret protooncogene and its sequence similarity to the cadherin superfamily. Oncogene 1993; 8: $1087-91$.

$\mathrm{m} / \mathrm{s} n^{\circ} 4$ vol. 10 , avril 94
19. Schneider R. The human protooncogene RET: a communicative cadherin. Trends Biochem Sci 1992; 17: 468-9.

20. Schuchardt A, D'Agati V, LarssonBlomberg L, Costantini F, Pachnis V. Defects ih the kidney and enteric nervous system of mice lacking the tyrosine kinase receptor Ret. Nature 1994; 367: 380-3.

21. Cass IT, Ling Z, Morthrope J. Aganglionosis in rodents. J Pediatr Surg 1992; 27: 351

22. Mulligan M, Kwok JBJ, Healey CS, Eldson MI, Eng C;, Gardner E, Love IDR, Mole SE, Moore JK, Papi L, Ponder MA, Telenius $\mathrm{H}$, Tunnacliffe $\mathrm{A}$, Ponder BAJ. Germline mutations of the $R E T$ proto-oncogene in multiple endocrine neoplasia type $2 \mathrm{~A}$. Nature 1993; 363: 458-60.

23. Donis-Keller H, Dou S, Chi D. Mutations in the RET proto-oncogene are associated with MEN 2A and FMTC. Hum Mol Genet 1993; 2: 851-6.

24. Mulligan L, Eng C; Healey CS, Clayton D, Kwok JBJ, Gardner E, Ponder MA, Frilling A, Jackon (E, Lehnert $\mathrm{H}$, Neumann HPH, Thibodeau SN, Ponder BAJ. Specific mutations of the RET protooncogene are related to disease phenotype in MEN 2A and FMTC. Nature Genet 1994; 6: $7(0-4$.

25. Hofstra RMW, Landsvater RM, Ceccherini I, Stulp RP, Stelwagen T, Luo Y, Pasini $B$, Hôppener JW'M, Ploos van Amstel HK, Romeo G, Lips C.JM, Buys C.HC'M. A mutation in the RET proto-oncogene associated with endocrine neoplasia type $2 \mathrm{~B}$ and sporadic medullary thyroid carcinoma. Nature $1994 ; 367: 375-6$.

26. Eng (;, Smith DP, Mulligan LM, Nagai MA, Healey C.S, Ponder MA, (iardner E, Schermann (BFW', Jacrson C.E, Tunnaclitte A, Ponder BJA. Point mutations within the tyrosine kinase domain of the $R E T$ proto oncogene in multiple endocrine neoplasia type 2B and related sporadic tumours. Hum Mol Genet 1994; 3:237-41.

\section{Stanislas Lyonnet, Patrick Edery, \\ Tania Attié, Claire Nihoul-Fékété, Amold Munnich et le Consortium Français de la Maladie de Hirschsprung \\ Service de génétique médicale, clinique chimergicale infantile et unité de recher- ches sur les handicaps génétiques de l'enfant, Inserm U. 393, hôpital des Enfants-Malades, 149, rue de Sèrres, 75743 Paris Cedex 15, France.}

\section{TIRÉS A PART}

S. Lyonnet. 